\title{
An Improved Ant Colony Optimization for VRP with Time Windows
}

\author{
Yu Su-Ping and Mao Wei-Wei \\ Computer and Information engineering department, Luoyang Institute of Science \\ and Technology, Luoyang, China \\ badkid@126.com; mw116@lit.edu.cn
}

\begin{abstract}
The Vehicle Routing Problem (VRP) is an important problem occurring in many distribution systems, which is also defined as a family of different versions such as the Capacitated Vehicle Routing Problem (CVRP) and the Vehicle Routing Problem with Time Windows (VRPTW). The Ant Colony Optimization (ACO) is a metaheuristic for combinatorial optimization problems. Given the ACO inadequacy, the vehicle routing optimization model is improved and the transfer of the algorithm in corresponding rules and the trajectory updated regulations is reset in this paper, which is called the Improved Ant Colony Optimization (I-ACO). Compared to the calculated results with genetic algorithm (GA) and particle swarm optimization (PSO), the correctness of the model and algorithm is verified. Experimental results show that the I-ACO can quickly and effectively obtain the optimal solution of VRFTW.
\end{abstract}

Keywords: VRP, CVRP, VRPTW, ACO, I-ACO

\section{Introduction}

The Vehicle Routing Problem (VRP) refers to a series of shipping point (or receiving point), form proper driving path, make the Vehicle orderly through them. While satisfying the constraint condition of cases, it can achieve a certain goal (such as the distance shortest, minimum cost, time consuming less as far as possible, use vehicles such as less as far as possible). VRP is a NP-hard problem, which is difficult to find the accurate solution, especially in the process of practical distribution. Due to more demand points and sections, this kind of problem is generally solved by a set of heuristic algorithm and evolutionary algorithm. With Internet popularizing and development of Electronic Commerce, the tradition VRP algorithm [1] fleetness already unable to cope with response (Quick Response) in time from customer's needs for logistics distribution, thereupon the concept of time window [2] arise. An important feature of distribution is that users tend to have time limit requirements of sending delivering, i.e., VRP problem with constraint of time Windows (VRPTW, the vehicle routing problem with time Windows). Suppose that there are $\mathrm{n}$ demand point for delivery, which expresses as $1, \ldots$, $\mathrm{n}$, demand point I has a fixed time of completion $\mathrm{Ti}$ and a service time $\mathrm{Si}$ (e.g., the unloading time). For example: If $\mathrm{Ti}=12: 30, \mathrm{Si}=1$ hour, and then the train must arrive in demand points $i$ at 11:30. DH $(i, j)$ is used to express the transport time between any two requirements $i, \quad j$ and dij as the distance. Assume that if $\mathrm{Tj}-\mathrm{Sj}>\mathrm{Ti}+\mathrm{DH} \quad(\mathrm{i}, \mathrm{j})$, and then $\operatorname{arc}(i, j)$ is existed. If $\operatorname{arc}(I, j)$ is specified cost $\mathrm{Cij}=\mathrm{DH}(\mathrm{i}, \mathrm{j})$ (or dij), thus, the problem is transformed into a given number path (specifying a sequence) from start $i$ to terminal $\mathrm{j}$, which passes all the nodes in order to minimise total transport time (or total transportation distance) in the schedules of all vehicles.

In actual distribution, it is fine if the distribution center deliver the goods within certain time range after receiving an order form (within 24 hours). The existence VRP of 
more vehicles is more but simple VRP and VRPTW or distribution of many dispatch centers is relatively less. Therefore, the thesis put an emphasis on the VRPTW distribution transporting problem; use the improved ant group algorithms to find the solution. Finding a feasible solution to the VRPTW is an NP-hard problem [3].

In particular, for the solution of VRP and its variations, a number of metaheuristics have been successfully applied, such as: simulated annealing [4], tabu search [5], granular tabu search [6], genetic algorithms [7], greedy randomized adaptive search procedure [8], and ACO [9]. The objective of this paper is to describe an I-ACO to solve the VRPTW with more competitive ability to obtain the optimal solution. On the basis of AOC, taking concrete requirement and constraint condition into consideration, making an improvement for choosing mechanism, renewing and coordination mechanism, leading into dynamic metastasis tactics and plain information renewal tactics, all is to overcome the defect of ant group algorithmic which is time-consuming and easy to be caught in part optimum solution.

\section{Design for Ant Group Algorithm}

Algorithm of symbols involved in stated below: $\mathrm{m}$ - ants number, ${ }^{i j}$-side $\operatorname{arc}(\mathrm{i}, \mathrm{j})$ on track intensity (intensity); $\eta_{i j}$-side $\operatorname{arc}((\mathrm{i}, \mathrm{j})$ on the visibility, take visibility (distance between nodes, namely, $1 / d_{i j}$; Ants $\Delta \tau_{i j}^{k}$ in side $\operatorname{arc}(\mathrm{i}, \mathrm{j})$ leaves of unit length track pheromone amount, The transition probability $p_{i j}^{k}$-ants k, $\alpha$-trajectory of the relative importance of ( $\alpha \geq 0$ ); $\beta$-the visibility of the relative importance of $(\beta \geq 0)$; $\rho_{\text {-track }}$ the permanence of $\left(0 \leq \rho \leq{ }_{1}\right),{ }_{1-} \rho$ understanding to track the volatilization of degrees (evaporation); $N_{i}^{k}$ — in the i nodes with ants k feasible vertex set, $\mathrm{Q}$ - reflect ants have kept track number of constant; $L^{k}$ - the first k ant tectonic path length.

\subsection{The Transferred Tactics of Algorithm}

Initial, an ant was put a random selected node, path structure is according to certain transition probability, the first $\mathrm{k}$ ant on $\mathrm{t}$ moment from node $\mathrm{i}$ to node $\mathrm{j}$ transfer probability for:

$$
p_{i j}^{k}(t)= \begin{cases}\frac{\left[\tau_{i j}(t)\right]^{\alpha}\left[\eta_{i j}\right]^{\beta}}{\sum_{l \in N_{i}^{k}}\left[\tau_{i l}(t)\right]^{\alpha}\left[\eta_{i l}\right]^{\beta}} & j \in N_{i}^{k} \\ 0 & \text { else }\end{cases}
$$

\subsection{The Renewed Tactics for Pheromone}

The concept of Selection Window is introduced before On the V (I) before discussing the application of $\mathrm{V}(\mathrm{i})$ in the algorithm transfer strategy.

Definition 3: Selection Window

Selection Window refers to the next node number of ant $\mathrm{k}$ that allows to select in the process of transfer, signified in Wink(i).

Which value is relatively proper for selection window setting? If the value is too small, even though it can achieve the purpose of reducing the scope of the search, the algorithm will be made into local solution; if too big, it will be ineffective and can not achieve the purposes of reducing the scope of the search and saving computation time. In addition, the value of selection window should be dynamically adjusted, as in the initial 
stage of the algorithm, in order to ensure global algorithm, selection window should be much larger. However, at some stage, the size of selection window should be adjusted based on the dynamically distributed solutions. The more concentrated solution, the larger selection window will be. On the contrary, the more dispersed solution is, the smaller selection window would be. In short, we hope that the selection window can not only make the algorithm to shorten the calculation time, but also does not have much impact on a global search. For this, we choose an integrated evenness V (i) to determine the size of the selection window ss.

The prior node of allowedk consists of the feasible point allowedk that ant $\mathrm{k}$ is revised in node $\mathrm{i}$, from which can easily know allowedk $\subseteq$ allowedk in order to effectively limit every move scope of these ants, reduce the search space of algorithm and save computational time.

According to track update Quantity of rules and update the time difference, the Ant colony algorithm is divided into three different versions: ants Density (Ant - Density) model, the population (Ant - Quantity) model and ants (Ant-Cycle) model. Among them, the ant density and the population model is whenever ants by point i move after, on tables $\mathrm{j}$ arc ( $\mathrm{i}, \mathrm{j}$ ) handling trajectory update (local update); But the ant laps model is in all the ants structure

Later, just finish path made for every arc handling trajectory updates. The ant laps model, its corresponding trajectories update quantity $\Delta t^{k}$ for:

$\operatorname{win}^{k}(i)=\left\{\begin{array}{l}\operatorname{cnt}\left(\text { allowed }_{k}\right) \\ i f(1-V(i)) * \operatorname{cnt}\left(\text { allowed }_{k}\right)=\text { cnt }\left(\text { allowed }_{k}\right) \\ {\left[(1-v(i)) * \operatorname{cnt}\left(\text { allowed }_{k}\right)\right]+1} \\ \text { else }\end{array}\right.$

Improved regulation is as follows:

$\tau_{i j}(t+n)=\rho \cdot \tau_{i j}(t)+\Delta \tau_{i j}(t, t+n)$

Its corresponding trajectories updated rules are as follows:

$\Delta \tau_{i j}(t, t+n)=\sum_{k=1}^{m} \Delta \tau_{i j}^{k}(t, t+n)$

\subsection{Design of a Negative Feedback Mechanism}

Selection mechanism of ant colony algorithm is to make the good path that will be selected with high probability, and the existing of positive feedback mechanism is bound to make these paths more competitive in the future selection. According to the traditional way, when searching fall into local optimal solution, the algorithm can not jump out of the local minimum points. For this, the negative feedback mechanism is introduced. It is comprised that a drawing of the MIN-MAX thought that the amount of information on each path is defined within a fixed range. To some extent, it is possible to reduce the gap between the concentrations of pheromone path, prompting algorithm jump out from the local minimum point. If there is stagnation, at the time of global updating, adding negative feedback information that lets Q2, Q3 $<0$, which can make local solutions reduce the amount of pheromone on the corresponding path.

\section{Algorithm Improvement Strategies}

The main title (on the first page) should begin $13 / 16$ inches ( 7 picas) from the top edge of the page, centered, and in Times New Roman 14-point, boldface type. Capitalize 
the first letter of nouns, pronouns, verbs, adjectives, and adverbs; do not capitalize articles, coordinate conjunctions, or prepositions (unless the title begins with such a word). Please initially capitalize only the first word in other titles, including section titles and first, second, and third-order headings (for example, "Titles and headings" — as in these guidelines). Leave two blank lines after the title.

Ant colony algorithm according to the basic rules of fixed mode updated information and determine route choice probability, ignore algorithm to search for the actual status, often produce accelerating convergence and prevent premature, stagnation phenomenon of conflict. So the method according to search state dynamic adjustment algorithm path selection strategy and information to refresh strategy, in accelerating convergence and prevent stagnation balance is necessary.

Use $\operatorname{win}^{k}(i)$ said ants $\mathrm{k}$ in node i carry transfer allows a selected node number. In the basic ant colony algorithm has $\operatorname{win}^{k}(i)=\operatorname{cnt}\left(\right.$ allowed $\left._{k}\right), \operatorname{cnt}\left(\right.$ allowed $\left._{k}\right)$ said the number of nodes in the collection. $\operatorname{win}^{k}(i)$ too small algorithm easily trapped into local solutions; If too big reach reduce search scope, saving calculation time purpose. Therefore shall the dynamic adjustment value of $\operatorname{win}^{k}(i)$, both to shorten the time of calculation and to global search does not produce greater impact. In this algorithm, the initial stage to guarantee the overall algorithm, a little bigger $\operatorname{win}^{k}(i)$,at some stage, according to the distribution of solutions for the size of $\operatorname{win}^{k}(i)$ the dynamic adjustment.

$$
\operatorname{win}^{k}(i)=\left\{\begin{array}{l}
\text { cnt }_{\left(\text {allowed }_{k}\right)} \\
\text { if }(1-V(i)) * \operatorname{cnt}\left(\text { allowed }_{k}\right)=\text { cnt }\left(\text { allowed }_{k}\right) \\
{\left[(1-v(i)) * \operatorname{cnt}\left(\text { allowed }_{k}\right)\right]+1} \\
\text { else }
\end{array}\right.
$$

Therefore using the comprehensive evenness V(i) to determine the size of $\operatorname{win}^{k}(i)$.

Meanwhile, in VRPTW, nodes allowed $_{k}$ should not only satisfy capacity constraints, also to meet time window constraint and renewal drive time constraint. Situated i point for allowed $_{k}$ :

allowed $_{k}=\left\{v_{j} \mid v_{j} \in V, g_{j}+\sum g \leq q, t_{i j}+\sum t \leq r_{k}, E T_{j} \leq s_{j} \leq L T_{j}\right\}$

Comprehensive evenness V(i) : set for a total of $\mathrm{M}$ ants, from node $\mathrm{i}$ were $\mathrm{r}$ path reached another $\mathrm{r}$ a quarter Point $i_{1}, i_{2}, \ldots, i_{r}$, the last time, i point after the iteration of the total is $\mathrm{Y}(\mathrm{i})$, wightii mosquitoes, and the $\mathrm{Y}(\mathrm{i})$ ant on $\mathrm{r}$ path distribution,respectively, $a_{1}, a_{2}, \ldots, a_{r}$, to remember

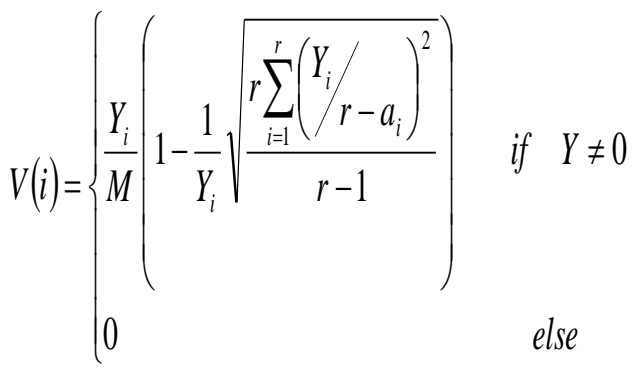


Improved metastasis regulation is as follows:

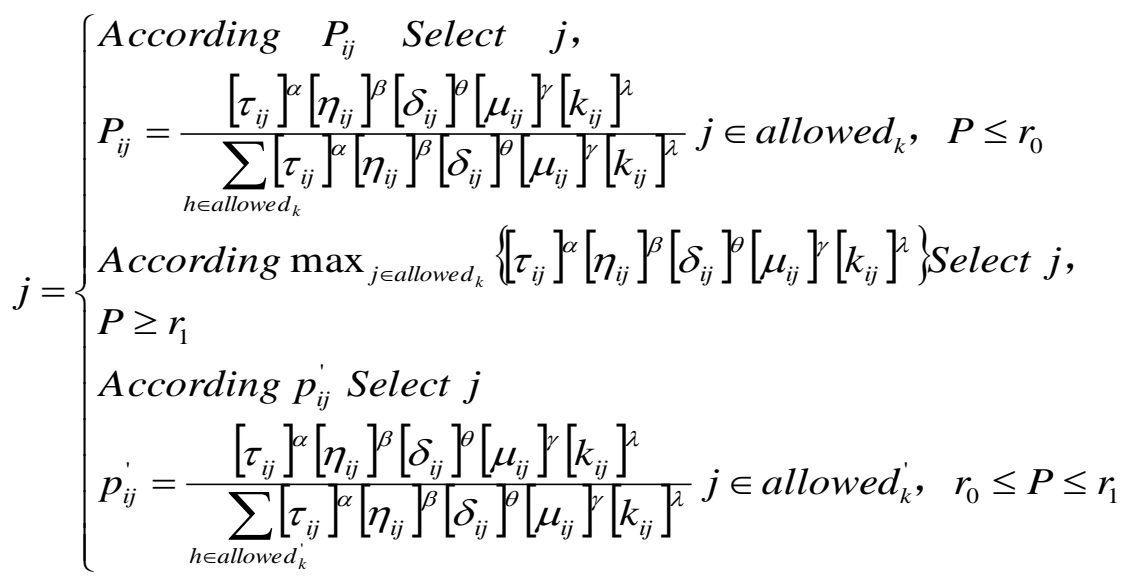

Among them, $\tau_{i j}$ expressing plain thickness pheromone edge (i,j), $\eta_{i j}$ expressing the visibility of edge $(\mathrm{i}, \mathrm{j})$, choosing the knot spacing reciprocal, that is $\eta_{i j}=1 / d_{i j}$. $\mu_{i j}=d_{i 0}+d_{0 j}-d_{i j}$. Taking in account that the variable from the distance from the task center to the distribution center room, we have to put importance on both the distance between point vi and point dij and the distribution positon between two points. $\mu_{i j}$ reflects the saving amount between the route of linking directly the two points and the route of respectively connecting two points with the distribution center. Obviously, when $\mu_{i j}$ is bigger, the profits is bigger, and the higher probability for choosing $\mathrm{vj}$. $k_{i j}=\left(Q_{i}+g_{i}\right) / Q$ is the variable when thinking that vehicle capacities restrain. $\mathrm{P}$ is a random number, obey $(0,1)$ siding-to-siding block. r1, r0 adjusted with algorithmic dynamic evolution. $\delta_{i j}$ Is considering time urgency and introduction of variable.

$$
\delta_{i j}=\quad 1 /\left(w_{1} \frac{\left|s_{i}+T_{i}+t_{i j}-E T_{j}\right|}{L T_{j}-E T_{j}}+w_{2} \frac{\left|s_{i}+T_{i}+t_{i j}-L T_{j}\right|}{L T_{j}-E T_{j}}\right)
$$

For the weight $w_{1}, w_{2}$ coefficient, and $w_{1}+w_{2}=1$. From the window that meets time constraint Angle of, the bigger $\delta_{i j}$ shows, choose j point more reasonable, $\theta$ is to decide $\delta_{i j}$ relative influence parameters.

\section{Analysis of Experimental Results}

According to the vehicle controller question with time window, the problem has 8 freights mission (serial number to be $1,2, \ldots, 8$ ), every mission volume of goods transported gi (unit: Ton), loading(or unloading) time Ti (unit: hour), as well as the time range of every item mission [ETi,LTi](unit: time) . all this is from the Table 1. all the missions are completed by vehicles that have capacity of 8 tons from 0 parking lot. The distance between Parking lot 0 every missionpoint (unit: Kilometre) is in Table 2. Speed of a vehicle 50, the unit transports cost 1 (unit: Yuan/ kilometre). 
Table 1. The Characteristics and Requirement OFTask

\begin{tabular}{|c|l|l|l|l|l|l|l|l|}
\hline Branch & 1 & 2 & 3 & 4 & 5 & 6 & 7 & 8 \\
\hline Requirement & 2 & 1.5 & 4.5 & 3 & 1.5 & 4 & 2.5 & 3 \\
\hline Time T & 1 & 2 & 1 & 3 & 2 & 2.5 & 3 & 0.8 \\
\hline Time window & {$[1,4]$} & {$[4,6]$} & {$[1,2]$} & {$[4,7]$} & {$[3,5]$} & {$[2,5]$} & {$[5,8]$} & {$[1,5]$} \\
\hline
\end{tabular}

Table 2. Branch Rooms and Branch and Distribution Center

\begin{tabular}{|c|l|l|l|c|l|l|l|l|l|}
\hline Distance & \multicolumn{1}{|c|}{0} & \multicolumn{1}{|c|}{2} & 3 & 4 & 5 & 6 & 7 & 8 \\
\hline 0 & 0 & 40 & 60 & 75 & 90 & 200 & 100 & 160 & 80 \\
\hline 1 & 40 & 0 & 65 & 40 & 100 & 50 & 75 & 110 & 100 \\
\hline 2 & 60 & 65 & 0 & 75 & 100 & 100 & 75 & 75 & 75 \\
\hline 3 & 75 & 40 & 75 & 0 & 100 & 50 & 90 & 90 & 150 \\
\hline 4 & 90 & 100 & 100 & 100 & 0 & 100 & 75 & 75 & 100 \\
\hline 5 & 200 & 50 & 100 & 50 & 100 & 0 & 70 & 90 & 75 \\
\hline 6 & 100 & 75 & 75 & 90 & 75 & 70 & 0 & 70 & 100 \\
\hline 7 & 160 & 110 & 75 & 90 & 75 & 90 & 70 & 0 & 100 \\
\hline 8 & 80 & 100 & 75 & 150 & 100 & 75 & 100 & 100 & 0 \\
\hline
\end{tabular}

Document [6] same use inheritance algorithm (GA) and particle group algorithm (PSO) two species method finds the solution, every runs 50 times. [6]'s experiment indicates the optimum algorithm parameter combination is $m=\sqrt{n} \sim n / 2, \quad \alpha=1 \sim 5$ $, \beta=1 \sim 5, \rho=0.7, \mathrm{Q}=100$. According to the conclusion, setting the the controlled parameter of algorithm: $\mathrm{M}=10, \mathrm{Ncmax}=60, \mathrm{rij}=10, \mathrm{MAX}=40, \mathrm{MIN}=2, \mathrm{r} 0=\mathrm{r} 1=1$. Find the solution and compared with the document, Table 4 shows as follows.

Table 3. The Optimization Results Compared

\begin{tabular}{|c|c|c|c|}
\hline Method & $\begin{array}{c}\text { Search } \\
\text { probability }\end{array}$ & $\begin{array}{c}\text { average } \\
\text { cost }\end{array}$ & $\begin{array}{c}\text { average successful } \\
\text { search time }\end{array}$ \\
\hline GA & $24 \%$ & 993.6 & 18.41 \\
\hline PSO & $46 \%$ & 940.5 & 8.53 \\
\hline I-ACO & $56.6 \%$ & 950.6 & 9.36 \\
\hline
\end{tabular}

In view of the above questions according to the soft time Windows problem solving, get transport 3 vehicles, optimal path for $780 \mathrm{~km}$, minimum cost for 815.7 yuan.

Experimental results show that the I - ACO of this issue in average successful search time and PSO almost, but search probability and driven an average cost has good performance. Especially search success rate than PSO improve nearly 10\%. Explain in this problem using this design I - ACO is ideal.

\section{Conclusion}

The thought of the thesis is: on the basis of AOC, taking concrete requirement and constraint condition into consideration, making an improvement for choosing mechanism, renewing and coordination mechanism, leading into dynamic metastasis tactics and plain information renewal tactics, all is to overcome the defect of ant group algorithmic which is time-consuming and easy to be caught in part optimum solution. 
Experimental results show that the I-ACO can quickly and effectively obtain the optimal solution of VRFTW.

Next problem demanding prompt solution is that how to decrease the difference between routes when the research sink into locally optimal solution in order to urge algorithm to jump from local minimum point; how to decrease information in the route of local solution if stasis phenomenon appears.

\section{Acknowledgments}

I would like to express my gratitude to all those who helped me during the writing of this thesis. My deepest gratitude goes first and foremost to the leaders in the Department of Computer and Information Engineering, who have instructed and helped me a lot during my work. I would like to express my heartfelt gratitude to my beloved family for their loving considerations and great confidence in me all through these years. I also owe my sincere gratitude to my friends who gave me their help and time in listening to me and helping me work out my problems during the difficult course of the thesis.

\section{References}

[1] C. D. Scott and R. E. Smalley, J. Nanosci. Nanotechnol, no. 75, vol. 3, (2003).

[2] I. H. Osman, "Metastrategy simulated annealing and tabu search algorithms for the vehicle routing problem," Annals of Operations Research, (1993).

[3] M. Gendreau, A. Hertz and G. Laporte, "A tabu search heuristic for the vehicle routing problem," Management Science, vol. 40, no. 10, (1994).

[4] P. Toth and D. Vigo, "The granular tabu search and its application to the vehicle routing problem," INFORMS Journal on Computing, vol. 15, no. 4, (2003), pp. 3462987.

[5] P. Toth and D. Vigo, "The Vehicle Routing Problem", Monographs on Discrete Mathematics and Applications, SIAM. Philadelphia, (2001).

[6] M. Fisher, "Vehicle routing", Handbooks of Operations Research and Management Science, (1995).

[7] A. V. Breedam, "An analysis of the effect of local improvement operators in genetic algorithms and simulated annealing for the vehicle routing problem," RUCA Working Paper 14, University of Antwerp, Belgium, (1996).

[8] M. G. C. Resende and C. C. Ribeiro, Greedy randomized adaptive search procedures, In F. Glover and G. Kochenberger (Eds.), Handbook of metaheuristics, Boston: Kluwer Academic, (2003).

[9] L. M. Gambardella, é. Taillard and G. Agazzi, "MACS-VRPTW: a multiple ant colony system for vehicle routing problems with time windows", In D. Corne, M. Dorigo and F. Glover (Eds.), New ideas in optimization, (1999).

\section{Authors}

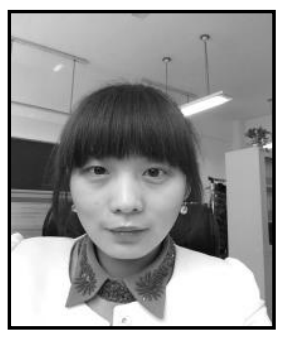

Yu Suping, Gender: Female, Professional title: Lecturer. Education: Master degree candidate of Henan University. Main research: Computer Application Technology

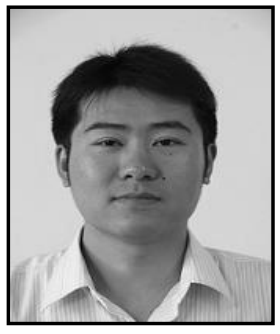

Mao Weiwei, Gender: Male, Professional title: Lecturer. Education: Master degree candidate of Sichuan University. Main research: Computer Application Technology. 
International Journal of Signal Processing, Image Processing and Pattern Recognition Vol. 9, No. 10, (2016) 